\title{
Probing the Polar Instability of Strained $\mathrm{SrTiO}_{3}$ with HAADF-STEM
}

Salva Salmani-Rezaie ${ }^{1}$, Kaveh Ahadi ${ }^{1}$ and Susanne Stemmer ${ }^{2}$

${ }^{1}$ University of California, Santa Barbara, Goleta, California, United States, ${ }^{2}$ University of California, Santa Barbara, Santa Barbara, California, United States

$\mathrm{SrTiO}_{3}$ is an incipient ferroelectric in unstrained, pure form, but easily becomes ferroelectric when subjected to small perturbations, such as mechanical stress or alloying. Understanding the nature of the ferroelectric transition in $\mathrm{SrTiO}_{3}$ is important as it can play an important role in other properties. For example, recently, it was found that the superconducting transition temperature is doubled in compressively strained $\mathrm{SrTiO}_{3}$ films [1,2] for which a ferroelectric transition precedes the superconducting state upon cooling. This points to the relationship between superconductivity and ferroelectricity where ferroelectricity of $\mathrm{SrTiO}_{3}$ can potentially be used to tune the nature of its superconducting state. $\mathrm{SrTiO}_{3}$ is believed to exhibit a prototype displacive, soft mode ferroelectric transition. A hallmark of order-disorder transitions, on the other hand, is the formation of polar domains above the Curie temperature. These polar regions percolate below the Curie temperature to form a longrange ordered ferroelectric state. Images of polar regions above the Curie temperature of ferroelectric $\mathrm{SrTiO}_{3}$ would provide the most direct evidence of an order-disorder transition. Atomic resolution highangle annual dark-field imaging in scanning transmission electron microscopy (HAADF-STEM) is a powerful technique that is capable of determining the atomic column positions with picometer precision and, consequently, can detect regions with subtle structural changes. Obtaining information on the columns with off-centered Ti columns from their non-polar positions sets up the premise for understanding the nature of the ferroelectric phase transition of epitaxially strained $\mathrm{SrTiO}_{3}$.

In this study, we use HAADF-STEM to measure Ti column displacement vectors in the compressively strained $\mathrm{SrTiO}_{3}$ films, which undergo a ferroelectric transition below $140 \mathrm{~K}$. $\mathrm{SrTiO}_{3}$ thin films were grown using hybrid molecular beam epitaxy (MBE) on (001) LSAT [( $\left.\mathrm{LaAlO}_{3}\right)_{0.3}\left(\mathrm{Sr}_{2} \mathrm{AlTaO}\right)_{0.7}$ and (001) $\mathrm{SrTiO}_{3}$ single crystals. To investigate the effect of Sm doping on the ferroelectricity of strained $\mathrm{SrTiO}_{3}$, we also studied the films with different carrier densities $\left(n 3 \mathrm{D}=6 \times 10^{19}, 1.4 \times 10^{20}\right.$ and $\left.2.8 \times 10^{20} \mathrm{~cm}^{-3}\right)$. The polarization vector was defined as the difference between the center of mass of the four surrounding $\mathrm{Sr}$ columns and the actual Ti-O column position obtained by a 2D Gaussian fitting.

Figure 1 highlights the formation of the polar domain in the paraelectric phase of the compressively strained $\mathrm{SrTiO}_{3}$ films, which undergoes a ferroelectric transition at the low temperature. Fig.1 (a-c) shows the representative HAADF-STEM image of $\mathrm{SrTiO}_{3}$ thin films on the $\mathrm{SrTiO}_{3}$ substrate and Fig. 1 (d-f) represents the images of strained $\mathrm{SrTiO}_{3}$ thin films grown on LSAT substrate. The magnitudes of the polarization vector for unstrained and strained $\mathrm{SrTiO}_{3}$ are depicted in Fig. 1(b) and (e) respectively. The arrows represent the directions of the polarization vectors and their magnitudes indicated by the color scale. The displacements in unstrained films are very small are a measure of experimental noise; in contrast, the displacements in the strained film are considerably large. Fig 1(c) and (f) show the direction of the displacement vector of the same region overlaid with the HAADF-STEM images for the un-strained and strained $\mathrm{SrTiO}_{3}$ films respectively. The color scale is based on the polarization direction with 30degree intervals. Whilst the Ti column displacement is random for the unstrained films, the Ti columns 
in the strained $\mathrm{SrTiO}_{3}$ film tend to displace in a favorable direction and hence form a nanodomain with the polarization direction of [001]. Figure 2 highlights the effect of doping and free charge carriers on the formation of polar domains in the strained and doped films. Sm dopants cause local lattice distortion in $\mathrm{SrTiO}_{3}$ films [3]. Color contour maps of Sm doped samples show the increase in lattice distortion caused by the Sm dopant ( $\sim 5 \%$ (a), $\sim 1 \%$ (b) and $\sim 2 \%$ (c)). The results show that the complex structural distortion caused by the Sm dopant atom shrinks the polar region size and leads to complete random distortion at a high concentration of Sm dopants [Fig 2(d) to (f)]. The highest doped $\mathrm{SrTiO}_{3}$ film (c and f) does not undergo the ferroelectric transition at low temperature, emphasizing the importance of the formation of the high-temperature ordered phase for the emergence of the ferroelectricity [4].
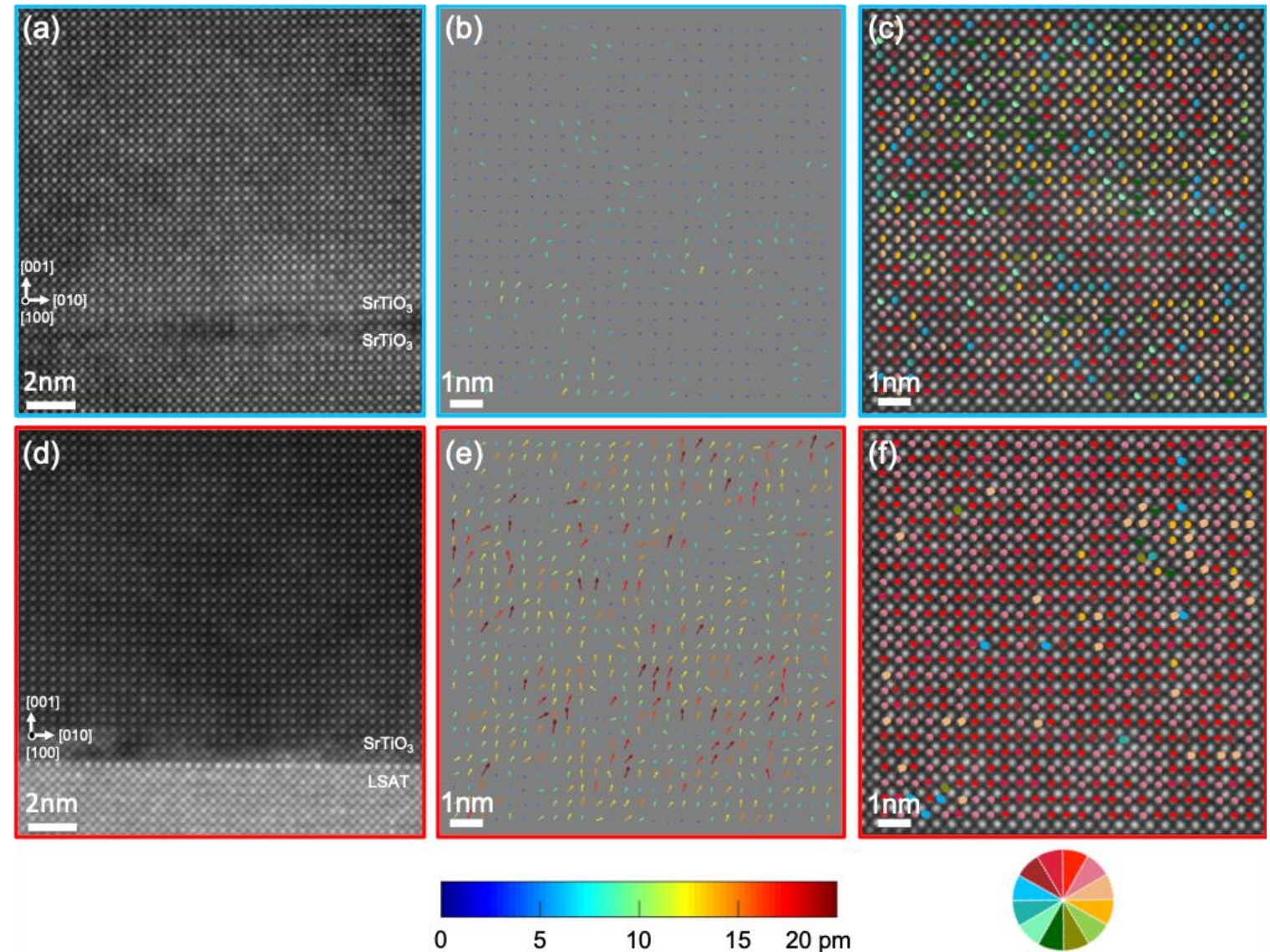

Figure 1. HAADF-STEM image of (a) unstrained and (b) strained SrTiO3. Polarization vectors for unstrained SrTiO3 (b, c) and strained (e, f) SrTiO3. The arrows in a and e indicate the magnitude of polarization. The images in the $\mathrm{c}$ and $\mathrm{f}$ display the direction of the polarization with each color corresponding to a 30-degree interval overlaid on the HAADF image 

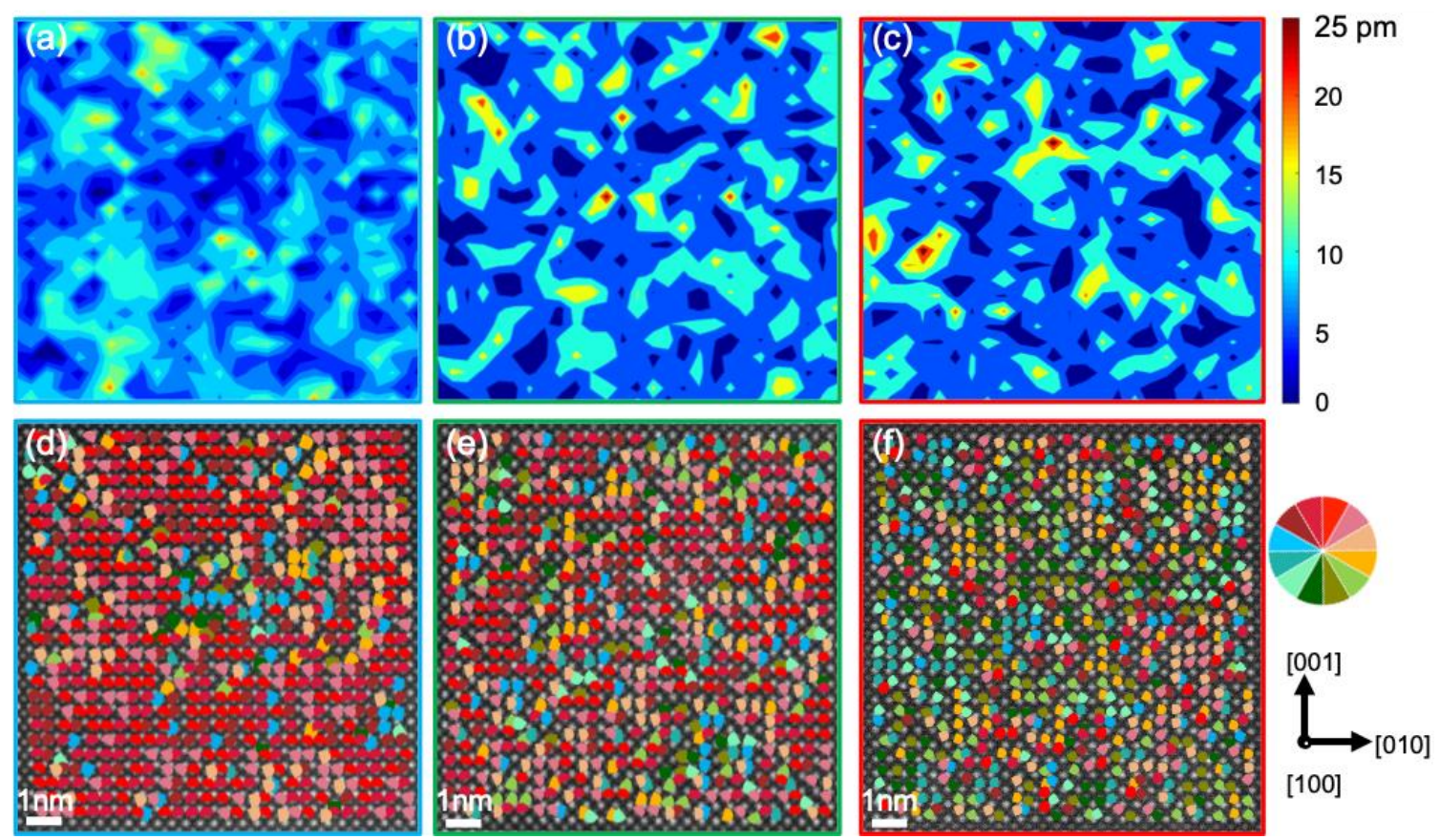

Figure 2. Color contour map (top) and polarization direction (bottom) for the SrTiO3 films with about $0.5 \% \mathrm{Sm}$ dopant ( a and d), $1 \% \mathrm{Sm}$ dopant (b and e), and 2\% Sm dopant (c and f)

\section{References}

[1] K. Ahadi, L. Galletti, Y. Li, S. Salmani-Rezaie, W. Wu, and S. Stemmer, Sci. Adv. 5, eaaw0120 (2019)

[2] R. Russell, N. Ratcliff, K. Ahadi, L. Y. Dong, S. Stemmer, and J. W. Harter, Phys. Rev. Mater. 3, 091401 (2019)

[3] S. Salmani-Rezaie, H. Kim, K. Ahadi, and S. Stemmer, Phys. Rev. Mater. 3, 114404 (2019)

[4] The authors acknowledge support by the U.S. Department of Energy (Grant No. DEFG02-2ER45994) 